\title{
Correction to: How Cross-Representational Signaling Affects Learning from Text and Picture: An Eye-Tracking Study
}

\author{
Juliette C. Désiron (D), Mireille Bétrancourt (D), \\ and Erica de Vries (D)
}

\section{Correction to: \\ Chapter "How Cross-Representational Signaling Affects Learning from Text and Picture: An Eye-Tracking Study" in: P. Chapman et al. (Eds.): Diagrammatic Representation and Inference, LNAI 10871, https://doi.org/10.1007/978-3-319-91376-6_68}

By mistake, the original version of this chapter was not published open access. The publishing mode has been changed to open access. 